\title{
EIT waves - A signature of global magnetic restructuring in CMEs
}

\author{
P. F. Chen and C. Fang \\ Department of Astronomy, Nanjing University, Nanjing 210093, China \\ email: chenpf@nju.edu.cn
}

\begin{abstract}
The discovery of "EIT waves" after the launch of SOHO spacecraft sparked wide interest among the coronal mass ejection (CME) community since they may be crucial to the understanding of CMEs. However, the nature of this phenomenon is still being hotly debated between fast-mode wave explanation and non-wave explanation. Accumulating observations have shown various features of the "EIT waves". For example, they tend to be devoid of magnetic neutral lines and coronal holes; they may stop near the magnetic separatrix between the source region and a nearby active region; they may experience an acceleration from the vicinity of the source active region to the quiet region, and so on. This paper is aimed to review all these features, discuss how these observations may provide constraints for the theoretical models, and point out their implication to the understanding of CMEs.
\end{abstract}

Keywords. Sun: CMEs, waves, Sun: UV radiation, Sun: activity, magnetic fields

After the launch in 1995 December, Solar and Heliospheric Observatory (SOHO) spacecraft (Domingo, Fleck, \& Poland 1995) provided unprecedented views of the structures and activities of the Sun, which greatly enhanced our understanding of the solar interior, solar atmosphere, solar wind, coronal mass ejections (CMEs), and so on. One of the most striking discoveries is the ubiquity of waves propagating in the low corona, conventionally referred to as "EIT waves", concurrent with the launch of front-disk CMEs as

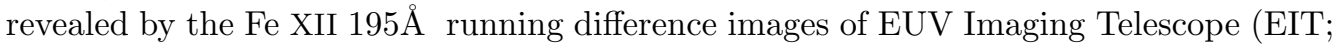
Delaboudinière et al. 1995) on SOHO. They not only manifest the low coronal signature of these more or less earth-directed CMEs, thus are very important for the space weather forecast, but also sparked hot debate of the relation between them and the Moreton waves discovered more than 40 years ago. This paper is aimed to piece the main properties of "EIT waves" together to see how they can be understood, and how the understanding can shed light on the nature of CMEs. $\S 1$ gives a general description of the "EIT waves", $\S 2$ reviews their properties which led to a fast-mode wave explanation, $\S 3$ is devoted to their properties which led to a non-wave explanation, and $\S 4$ summarizes how the diversity of their features can fit into a self-consistent picture, followed by a discussion of the implication of the understanding of "EIT waves" to the nature of CMEs in $\S 5$.

\section{Introduction}

"EIT waves" are often observed as almost circular diffuse emission enhancements (ranging from $25 \%$ to less than $14 \%$ ) propagating across the whole solar disk immediately followed by an expanding dimming region when the magnetic structure on the Sun is simple with only one active region on the disk (Thompson et al. 1998), as shown by figure 1. While, when the global magnetic structure gets complicated, they propagate rather inhomogeneously, avoiding strong magnetic features and neutral lines, as pointed 


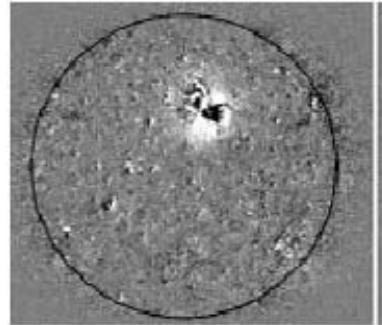

04:50 UT - 04:34 UT

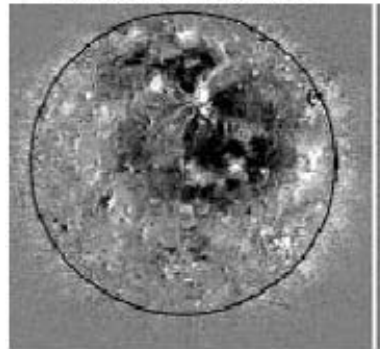

05:24 UT - 05:07 UT

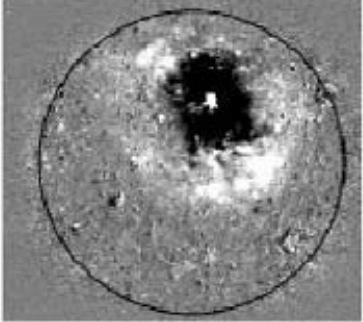

05:(07 UT - 04:50 UT

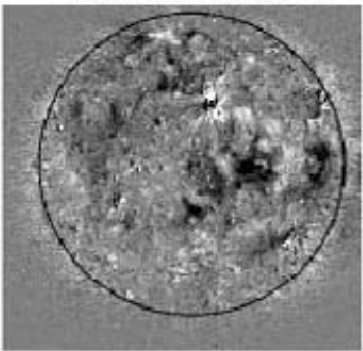

05:41 UT - 05:24 UT

Figure 1. An example of an "EIT wave" event showing almost circular fronts when the magnetic structure on the Sun is simple (Thompson et al. 1998).

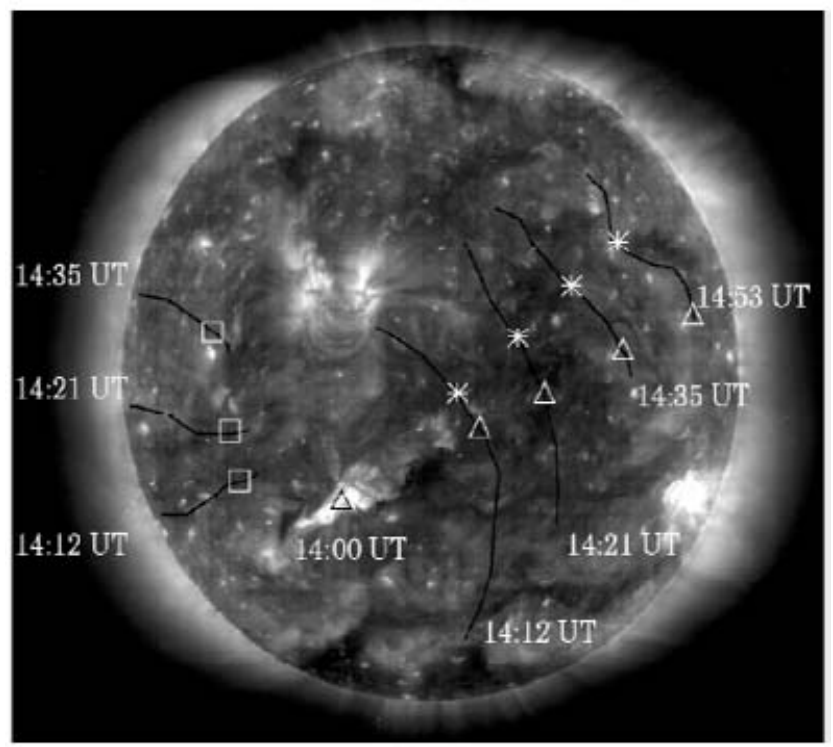

Figure 2. An example of an "EIT wave" event where fronts are propagating inhomogeneously when there are several active regions on the Sun (Thompson et al. 1999).

out by Thompson et al. (1999), who also found that "EIT waves" generally stop near coronal holes, as depicted in figure 2.

A careful examination by Biesecker et al. (2002) revealed that there is always a oneto-one correspondence from "EIT waves" to CMEs, though the contrary is not true. Their correlation with solar flares is significantly weaker, and they frequently are not accompanied by type II radio bursts (note that at least $90 \%$ type II bursts are associated with "EIT waves", as pointed out by Klassen et al. 2000). Therefore, "EIT waves" are 
a phenomenon intrinsically connected with CMEs. The speeds of these "EIT waves"associated CMEs range from $\sim 200-1800 \mathrm{~km} \mathrm{~s}^{-1}$, and there is no correlation between the occurrence of an "EIT wave" and the linearly fitted speed of the CME (Kay et al. 2003).

The statistical study by Klassen et al. (2000) indicates that the typical velocities of "EIT waves" range from 170 to $350 \mathrm{~km} \mathrm{~s}^{-1}$, with a mean velocity of $271 \mathrm{~km} \mathrm{~s}^{-1}$. Velocity as low as $80 \mathrm{~km} \mathrm{~s}^{-1}$ has also been reported (Dere et al. 1997). Roughly these values are three or more times smaller than the speeds of chromospheric Moreton waves (Moreton \& Ramsey 1960). The start times of "EIT waves" seem to be earlier than the type II radio bursts when they are associated, and be roughly concurrent with the associated type III bursts, which can serve as the flare onset reference.

It is noted that some telescopes other than $S O H O / E I T$ can also observe this wave phenomenon. For example, TRACE spacecraft captured several "EIT wave" events in both 171 and $195 \AA$ bands with better cadence but narrower field of view than $S O H O / \mathrm{EIT}$ (e.g., Wills-Davey \& Thompson 1999). Besides, He I waves cospatial with "EIT waves" are observed by CHIP telescope at Mauna Loa via He I $10830 \AA$ line, the formation of which is strongly affected by the thermal parameters in the low corona (Gilbert et al. 2004).

\section{Properties leading to the wave explanation and relevant modeling}

More than 40 years ago, $\mathrm{H} \alpha$ off-band observations of some big flare events showed that a kind of chromospheric disturbances propagate from the flare site to distances on order of $5 \times 0^{5} \mathrm{~km}$ with a velocity ranging from 500 to $2000 \mathrm{~km} \mathrm{~s}^{-1}$, which were later called Moreton waves (Moreton \& Ramsey 1960). Since they could not be any wave of chromospheric origin, Uchida $(1968,1974)$ then proposed that the skirt of the wave front surface of a coronal fast-mode wave or even shock wave sweeps the chromosphere, and produces the Moreton waves. The shocked coronal wave is also the source region of the associated type II radio bursts. According to this self-consistent model, there should exist the coronal counterparts of the chromospheric Moreton waves, which could be observed in soft X-ray or EUV wavelengths.

EUV emission of the Sun comes mainly from the transition region and low corona up to heights of $0.15 R_{\odot}$. Since "EIT waves", originating from the source active region, propagate across the quiet regions where the magnetic field lines are more or less radial, with a mean velocity significantly above the sound speed in the low corona (i.e., $\sim 179 \mathrm{~km}$ $\mathrm{s}^{-1}$ ), they are widely believed to be fast-mode magnetoacoustic waves, i.e., the coronal counterparts of $\mathrm{H} \alpha$ Moreton waves (e.g., Klassen et al. 2000). The belief was strengthened by the fact that in some "EIT wave" events there is a sharp wave front which is cospatial with almost simultaneous Moreton wave front (Thompson et al. 2000b) as seen in figure 3, though the "EIT wave" speeds are statistically three or more times smaller than Moreton wave speeds. Regarding to this incompatibility, Warmuth et al. (2001, 2004) noticed that Moreton waves are visible only near the flare sites, while "EIT waves" are mostly observed at larger distances. Therefore, they postulated that there might be a deceleration during the wave propagation.

The wave hypothesis is tested by the model simulation of Wang (2000) and 3D numerical simulations of Wu et al. (2001) and Li, Zheng, \& Wang (2002). All of them found that the propagation of fast-mode wave can match the observed "EIT wave" fronts, keeping in mind that all of them used the large-scale magnetograms of the Wilcox Solar Observatory to extrapolate the coronal potential field. As for the velocity discrepancy between EIT and Moreton waves, Wang (2000) postulated that the former could be ordinary waves, while the latter be super-Alfvén wave shock waves. 


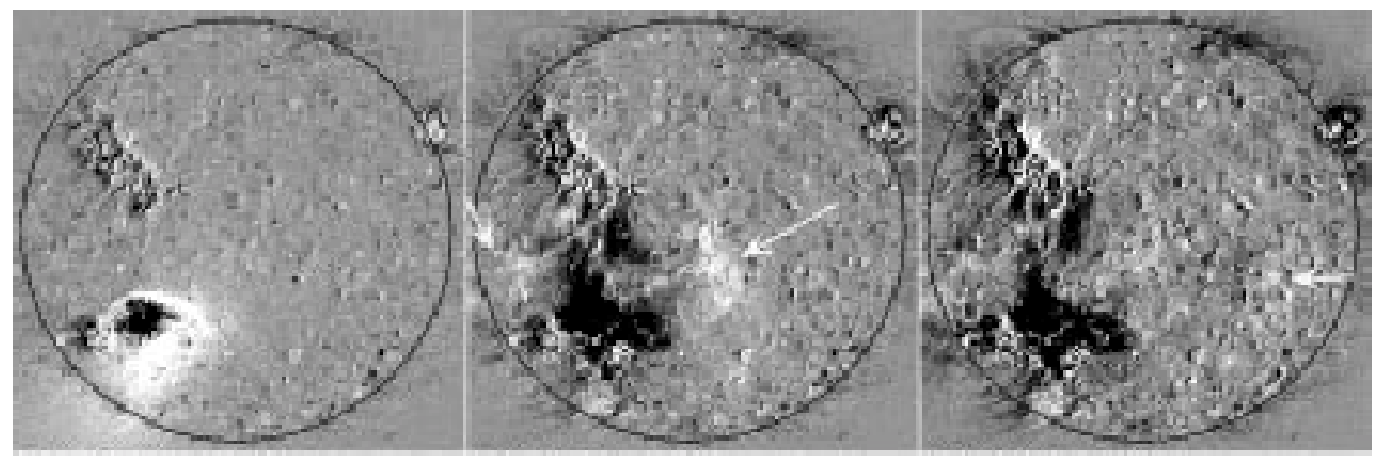

Figure 3. An example of an "EIT wave" event where one sharp bright EUV front is cospatial with the $\mathrm{H} \alpha$ Moreton wave (Thompson et al. 2000b).

\section{Properties leading to a non-wave explanation and relevant modeling}

Straightforward evidence to support non-fast mode wave explanations is the velocity discrepancy. Different from the proposal of Warmuth et al. (2001, 2004), Foley et al. (2003) found in an event that the "EIT waves" experience an acceleration rather than deceleration from the vicinity of the source active region to a large distance in the quiet region. Moreover, it is inferred from a winking filament by Eto et al. (2002) that the Moreton waves propagate much ahead of the "EIT waves" without deceleration at a large distance.

In the apparent appearances, it is noted by Thompson et al. (1999) that the relatively weak amplitudes and the diffuse fronts of the "EIT waves" indicate that they are not always be shocklike in nature. Another important point is that "EIT waves" often have circular shapes, while Moreton waves rarely span an angle of more than $160^{\circ}$. In the relation with type II radio bursts, it was well established that the speeds of the radio bursts exceed, but are typically proportional to, the associated Moreton wave velocities (Pinter 1977). In contrast, the recent statistical study by Klassen et al. (2000) pointed out that the radio burst speeds are not correlated with the "EIT wave" speeds, which also indicates that the "EIT waves" are not the coronal counterparts of the H $\alpha$ Moreton waves. More serious doubt comes from the discovery of a stationary "EIT wave" front in some events by Delannée \& Aulanier (1999) and Delannée (2000) (as shown by figure 4), who found that the "EIT waves" propagate initially, and then stop just near the footpoints of the magnetic separatrix, a characteristic far different from that of a magnetohydrodynamic wave. They suggested that the "EIT waves" should be related to the rearrangement of the magnetic structure in CMEs, where the bright fronts are due to the compression during the opening process of the magnetic structure.

There is some other indirect evidence to support that the "EIT waves" are not the coronal Moreton waves. For example, assuming that the shocked coronal Moreton waves are the acceleration site of energetic particles associated with CMEs, Bothmer et al. (1997) and Krucker, Larson, \& Lin (1999) compared the "EIT wave" positions and the footpoint of the Parker spiral connecting the Sun to Earth along which energetic particles are transported, and found that the diffuse "EIT waves" are two times slower to propagate from the flare site to the particle acceleration site (or the coronal Moreton wave front).

By performing numerical simulation of the eruption of CMEs, Chen et al. (2002) found that two types of wave structures are associated with CMEs. One is the pistondriven shock straddling over the erupting flux rope and sweeping the solar surface with a 


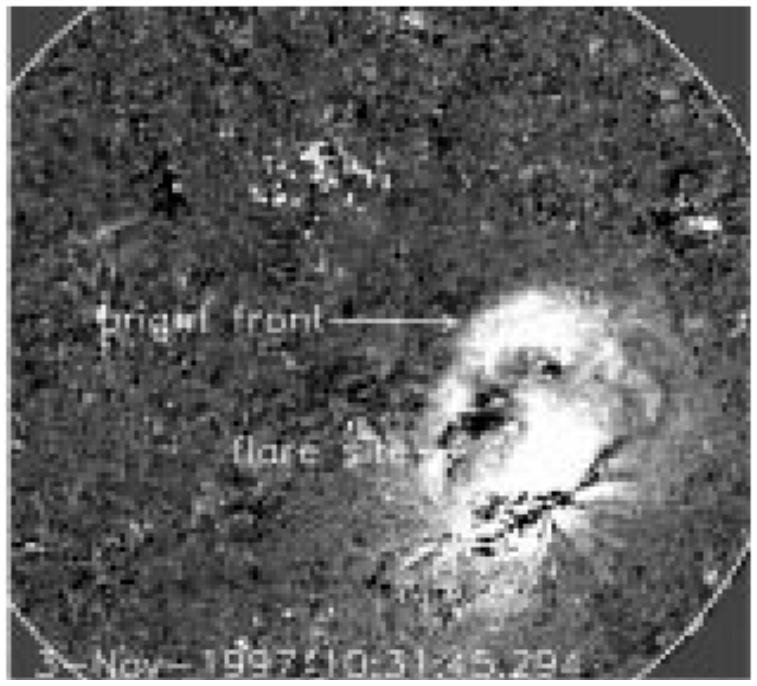

Figure 4. An example of an "EIT wave" event where the propagating "wave" stops near the footpoint of magnetic separatrix to form a stationary front (Delannée \& Aulanier 1999).

super-Alfvén speed, which they proposed corresponds to the coronal counterparts of H $\alpha$ Moreton waves; the other wavelike structure appears behind the coronal Moreton wave as propagating density enhancements, followed by an expanding dimming region, with a speed about three times smaller, which they proposed corresponds to the observed "EIT waves", as shown by figure 5. They further put forward a theoretical model for the phenomenon, which is sketched in figure 6, where "EIT waves" are thought to be formed by successive opening of magnetic field lines covering the erupting flux rope, and therefore, they are not real waves. The model self-consistently explained the relationship between "EIT waves" and EIT dimming, and solved the velocity discrepancy between "EIT waves" and Moreton waves. A further consideration of the model indicated that the thickness of "EIT wave" fronts increases as they propagate, and it would be comparable to the size of the dimming region behind it (Chen \& Shibata 2002). This model was strongly supported by the delicate observational analysis by Harra \& Sterling (2003), who found that two waves emanate from the flare site with different propagation velocities, and the Doppler motions are significant only behind the slower wave.

\section{A full view of "EIT waves"}

As pointed out by Biesecker \& Thompson (2002), there is confusion in the literature as to what an "EIT wave" is, and multi-wavelength observations indicate that two types of waves are associated with CMEs, which correspond to the classical Moreton wave and to what we call the "EIT wave". Several factors are responsible for the long-standing confusion. The first is that the time cadence of EIT instrument is $\sim 15 \mathrm{~min}$, which makes it difficult for the fast moving coronal Moreton wave to be detected in at least two consecutive images. Though TRACE spacecraft has a much higher cadence, its field of view at $195 \AA$ is a little small. The second factor is that there are far less $\mathrm{H} \alpha$ Moreton wave observations than "EIT waves". Besides, the velocities of "EIT waves" and Moreton waves overlap around $300-400 \mathrm{~km} \mathrm{~s}^{-1}$, hence, it is hard to tell whether some events are high-speed "EIT waves" or low-speed coronal Moreton waves in EIT images. Even though, the hints of the existence of two types of EUV waves, i.e., coronal Moreton wave 


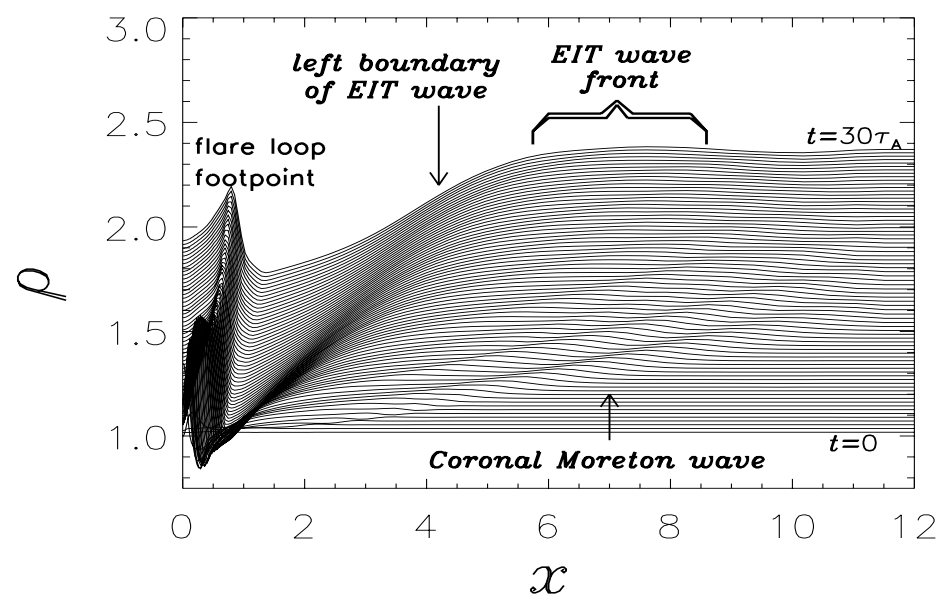

Figure 5. Numerical results showing a wavelike structure propagating behind the coronal Moreton wave with a velocity about three times smaller than coronal Moreton wave (Chen et al. 2002).

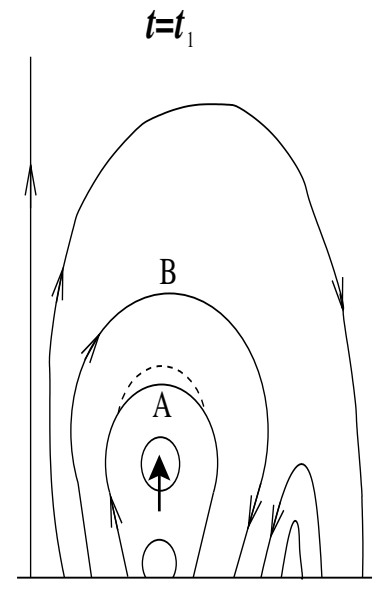

C D

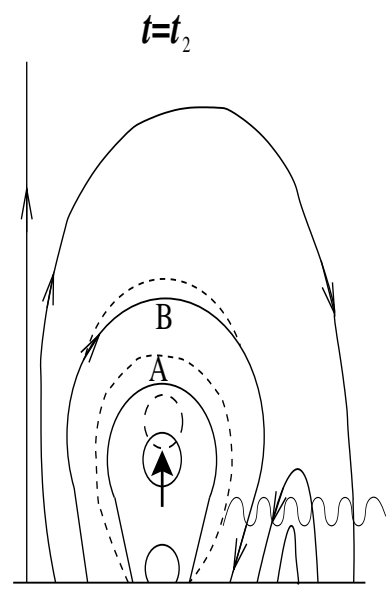

C D

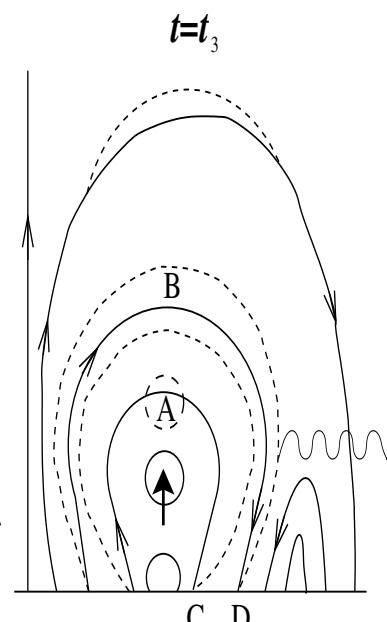

C D

Figure 6. Sketch map showing the propagation of the EIT wave front from point $\mathrm{C}$ to point $\mathrm{D}$, where the rising flux rope is the original source for the successive opening of all field lines. Solid lines represent the initial configuration, dashed lines correspond to the new configuration at each new time, while wave lines represent the fast-mode waves emitted from the EIT wave fronts. Moreton waves, much ahead of the EIT wave fronts, are not shown. (Chen et al. 2002).

and "EIT wave", distribute widely in the literature. Recently, Chen, Fang, \& Shibata (2004) summarized the different observational characteristics of the two types of waves detectable in EUV images, and discussed how these properties can fit the model proposed by Chen et al. (2002):

(1) The coronal Moreton wave, like the first EUV wave front cospatial with $\mathrm{H} \alpha$ Moreton wave in Thompson et al. (2000b), appears as a sharp, extremely bright feature; In contrast, the "EIT wave" fronts are rather diffuse and faint. This is because the coronal Moreton wave is a shock wave in nature, while the "EIT wave" corresponds to propagating large-amplitude perturbations formed by successive opening of closed field lines; 
(2) Several fronts of the "EIT wave" may be captured in each event, while at most only one front of the coronal Moreton wave, which may have also been seen as the soft X-ray wave (Khan \& Aurass 2002; Hudson et al. 2003), can be detected by SOHO/EIT in most "EIT wave" events owing to the low cadence. Only when the coronal Moreton wave has a speed near its lower limit, i.e., $\sim 300-400 \mathrm{~km} \mathrm{~s}^{-1}$, several EUV fronts can be found to be cospatial with $\mathrm{H} \alpha$ Moreton waves in a single event. Some of this kind of events were probably analyzed by Gilbert et al. (2004), Okamoto et al. (2004, the second event in their Table 1), and Vršnak et al. (2002);

(3) The coronal Moreton wave tends to propagate in a narrow direction where the magnetic field is weak owing to wave refraction. In contrast, a lot of "EIT waves" propagate almost circularly as all the field lines covering the flux rope are pulled up to open. They are devoid of magnetic neutral lines since no field lines are rooted there; they stop near magnetic separatrix, either between two active regions or near the boundary of coronal holes, to form a stationary front since the field lines outside the separatrix do not cover the erupting flux rope, and therefore do not open;

(4) The velocities of Moreton waves are almost proportional to those of type II radio bursts, since these two phenomena have the same driving agent, i.e., the piston-driven shock straddling over the CME. In contrast, there is a lack of correlation between the velocities of "EIT waves" and those of type II bursts since the former are determined by both the magnetic field strength and the magnetic geometry;

(5) $\mathrm{H} \alpha$ Moreton waves experience little deceleration during their propagation, while "EIT waves" may experience an acceleration from the vicinity of the source active region to quiet region far away (e.g., Foley et al. 2003). The smaller speed of the "EIT waves" near the active region is strongly against the wave explanation, and is accounted for by the stretched magnetic configuration in our model;

(6) Moreton wave fronts and their wake are not associated with strong Doppler motions, while the "EIT waves" are followed by an expanding dimming region where substantial Doppler motions are observed (Harra \& Sterling 2001, 2003). This is because "EIT waves" are not oscillating waves. They are formed by successive opening of field lines covering the erupting flux rope, therefore, correspond to the propagation of largeamplitude perturbations with substantial Doppler motions.

\section{Implication to CMEs and a remark}

One of the intriguing questions about the onset of CMEs is how to connect relatively small-scale activity in the lower corona (e.g., solar flares) to the large-scale, often global, CME structures that are observed in the outer corona. As pointed out by Plunkett et al. (2002), "EIT waves" may be the means by which this cross-scale connection is established. After the onset of a CME, "EIT waves" propagate outward from the flare site to a large distance, immediately followed by an expanding dimming region. It was found that the EIT dimming region map the footprint of the CME (Thompson et al. 2000b; Harrison et al. 2003). Considering the spatial roughness and the nature of the two phenomena, we propose that it is the "EIT waves" that map the footprint of the CME leading edge, and the dimming region maps the the bottom of the CME cavity. Therefore, we extend the model sketch for the typical CMEs of Forbes (2000) to include "EIT waves" and EIT dimming as shown in figure 7 . Along this line of thought, it is tentatively suggested that CME leading edges and cavities have the same formation mechanism as "EIT waves" and EIT dimmings, i.e., they are formed by successive opening of field lines covering the erupting flux rope, which was proposed by Chen et al. (2002). In a word, "EIT wave" fronts correspond to the lower legs of CME leading edges near the solar surface. 


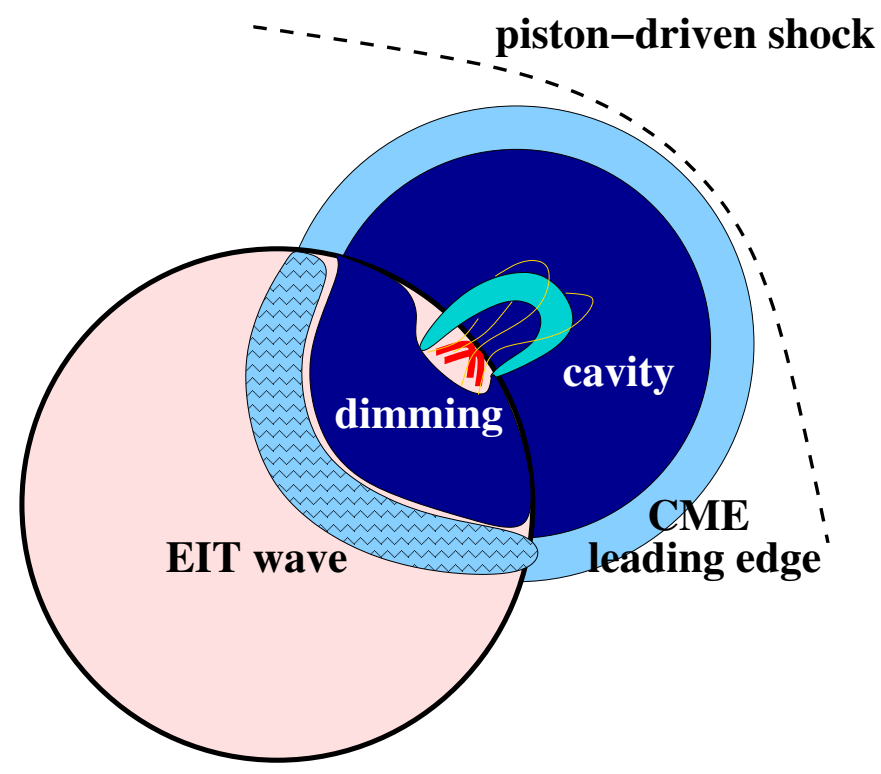

Figure 7. A sketch of our model indicating the relationship between CMEs and EIT waves/dimming.

Finally, a remark is made regarding the occurrence of "EIT waves". It has long been noticed that EIT dimmings occur much more frequently than "EIT waves". Similarly, Biesecker et al. (2002) pointed out that "EIT waves" are always associated with CMEs, which is consistent with our model that "EIT waves" are generated by successive opening of field lines in CMEs, while many CMEs have no associated "EIT waves", though largescale magnetic rearrangement always occurs in CMEs. Chen, Fang, \& Shibata (2004) suggested that the magnetic geometry could contribute to it since the compression outside the opening field lines is significant when the field lines are more or less perpendicular to the solar surface. If the field lines are strongly divergent outward as the initial condition in Chen \& Shibata (2000), the compression outside the opening field lines is substantially weak, therefore, the resulting "EIT wave" fronts would be too faint to be detected. Besides the geometry effect, we emphasize here the role of temperature variation, since it is often assumed implicitly that "EIT waves" are purely due to the density enhancement. In fact, however, a 10\% increase of the plasma density would be associated with an $7 \%$ increase of the plasma temperature in an adiabatic process. It is noted that the line emission contribution function for the Fe XII $195 \AA$ is sensitive to the temperature around $1.4 \times 10^{6} \mathrm{~K}$. The $\sim 7 \%$ increase of the plasma temperature would result in an $\sim 17 \%$ decrease of the emissivity, which may explain the absence of "EIT waves" in many CME events. Or, the occurrence of the "EIT waves" favors the low corona environment with a temperature a little lower than $1.4 \times 10^{6} \mathrm{~K}$, where the line emission contribution function increases with an increasing temperature.

\section{Acknowledgements}

The research is supported by FANEDD (200226), NSFC (10221001, 10333040 and 10403003) and NKBRSF (G20000784). 


\section{References}

Biesecker, D. A., Myers, D. C., Thompson, B. J. et al. 2002, ApJ 569, 1009

Biesecker, D. A. \& Thompson, B. J. 2002, BAAS, 34, 695

Bothmer, V. et al. 1997, in: A. Wilson (ed.), Proc. 31st ESLAB Symposium (Noordwijk: ESA), vol. (SP-415), p. 207

Chen, P. F., Fang, C., \& Shibata, K. 2004, ApJ, submitted

Chen, P. F. \& Shibata, K. 2000, ApJ 545, 524

Chen, P. F. \& Shibata, K. 2002, in: S. Ikeuchi, J. Hearnshaw, \& T. Hanawa (eds.), Proc. of the IAU 8th Asian-Pacific Regional Meeting, vol. II, p. 421

Chen, P. F., Wu, S. T., Shibata, K., \& Fang, C. 2002, ApJ (Letters) 572, L99

Delaboudiniére, J.-P. et al. 1995, Solar Phys. 162, 291

Delannée, C. 2000, ApJ 545, 512

Delannée, C. \& Aulanier, G. 1999, Solar Phys. 190, 107

Dere, K. P., Brueckner, G. E., Howard, R. A. et al. 1997, Solar Phys. 175, 601

Domingo, V., Fleck, B., \& Poland, A. I. 1995, Solar Phys. 162, 1

Eto, S., Isobe, H., Narukage, N. et al. 2002, PASJ 54, 481

Foley, C. R., Harra, L. K., Matthews, S. A., Culhane, J. L., Kitai, R. 2003, A\&A 399, 749

Forbes, T. G. 2000, J. Geophys. Res. 105, 23153

Gilbert, H. R., Holzer, T. E., Thompson, B. J., \& Burkepile, J. T. 2004, ApJ 607, 540

Harra, L. K. \& Sterling, A. C. 2001, ApJ(Letters) 561, L215

Harra, L. K. \& Sterling, A. C. 2003, ApJ 587, 429

Harrison, R. A., Bryans, P., Simnett, S. M., \& Lyons, M. 2003, A\& A 400, 1071

Hudson, H. S., Khan, J. I., Lemen, J. R., Nitta, N. V., \& Uchida, Y. 2003, Solar Phys. 212, 121

Kay, H. R. M., Harra, L. K., Matthews, S. A. et al. 2003, A\&A 400, 779

Khan, J. I. \& Aurass, H. 2002, A\&A 383, 1018

Klassen, A., Aurass, H., Mann, G., \& Thompson, B. J. 2000, A\& A Suppl. 141, 357

Krucker, S., Larson, D. E., \& Lin, R. P. 1999, ApJ 519, 864

Li, B., Zheng, H.N. \& Wang, S. 2002, Chinese Phys. Lett. 19, 1639

Moreton, G. E. \& Ramsey, H. E. 1960, PASP 72, 357

Okamoto, T. J., Nakai, H., Keiyama, A. et al. 2004, ApJ 608, 1124

Plunkett, S. P., Michels, D. J., Howard, R. A. et al. 2002, Adv. Space Res., 29, 1473

Thompson, B. J., Cliver, E. W., Nitta, N. et al. 2000a, GRL 27, 1431

Thompson, B. J., Gurman, J. B., Neupert, W. M. et al. 1999, ApJ(Letters) 517, L151

Thompson, B. J., Plunkett, S. P., Gurman, J. B. et al. 1998, Geophys. Res. Lett. 25, 2465

Thompson, B. J., Reynolds, B., Aurass, H. et al. 2000b, Solar Phys. 193, 161

Uchida, Y. 1968, Solar Phys. 4, 30

Uchida, Y. 1974, Solar Phys. 39, 431

Vršnak, B., Warmuth, A., Brajša, R., \& Hanslmeier, A. 2002, A\&A 394, 299

Wang, Y. -M. 2000, ApJ(Letters) 543, L89

Warmuth, A., Vrsnak, B., Aurass, H., \& Hanslmeier, A. 2001, ApJ(Letters) 560, L105

Warmuth, A., Vršnak, B., Magdalenić, J., Hanslmeier, A., \& Otruba, W. 2004, A\&\&A 418, 1101

Wills-Davey M. J. \& Thompson, B. J. 1999, Solar Phys. 190, 467

Wu, S. T., Zheng, H. N., Wang, S. et al. 2001, J. Geophys. Res. 106, 25089

\section{Discussion}

KAHLER: Do you expect to see a correlation between EIT wave speeds and associated CME speeds in your model?

Chen: Not for the leading edge speeds of CMEs. There may be a correlation between EIT wave speeds and the expansion speeds of the CME footpoints, which in fact map into the EIT wave front. 
SLEMZIN: In your model of coronal wave, do the waves exist in the transition region or not?

Chen: They should exist there, while the strength of the wave front will rapidly decrease as the formation height of the emission line decreases.

Kouтснму: I am wondering how important is gravity in your simulations? I see a good reason to have a faster speed (velocity): they propagate horizontally without work produced against gravity, which is not the case of EIT (coronal) waves!

Chen: We did not include gravity for the current simulations. The important thing regarding the velocity discrepancy might be their different mechanisms for the two kinds of waves. The coronal counterpart of Moreton waves are fast-mode (shock) waves in nature, while EIT waves are formed by successive opening the closed field lines covering the erupting flux rope. The role of gravity is not so important, I suppose.

ZHUKOv: I have two comments: one to support your model and the second is against it. First, any interpretation of EIT waves as fast-mode wave - regardless of deceleration (as suggested by Warmeth et al., or presence of a shock - has the plasma beta in the quiet Sun more than 1. This assumption is doubtful. Second, in your model EIT wave propagates together with a dimming as a result of successive opening of field lines. However, in some events (e.g. May 12, 1997) the EIT wave propagates to longer distances than the dimming.

Chen: Yes, the plasma beta in a major part of the lower corona might be much smaller than unity. As for your second comment, I should say that the EIT wave front is quite diffuse, with large width, which was explained by Chen \& Shibata (2002).

JUN LIN: How fast is the CME associated with the EIT wave in your simulation?

Chen: A little larger than the Moreton wave speed, i.e., about $800 \mathrm{Km} / \mathrm{s}$, which is about 3 times faster than EIT waves. 